Abb. 1 zeigt die Resultate für die einfachsten Fälle. In einer Platte von Ge (Dicke $1 \mathrm{~mm}$ ) mit (111)-Oberflächen entsteht durch eine (220)-Reflexion von $\mathrm{CuK} \alpha_{1}$ Strahlen ein Strahlenfächer, der senkrecht zur Oberfläche liegt (symmetrischer LaUe-Fall). Eine Stufenversetzung liegt parallel zur Oberfläche und senkrecht zum Strahlenfächer. Der Burgers-Vektor $\frac{1}{2}\langle 110\rangle$ liegt senkrecht zur reflektierenden (220)-Netzebene. Wegen der Symmetrie wird nur die rechte Hälfte des Fächers gezeigt. Es sind nur einige der berechneten Strahlen eingezeichnet, die eine merkliche Krümmung haben. Um die Verbiegung zu verdeutlichen, wurde der Maßstab der Abszisse 10-fach vergrößert. Die Strahlen ge-

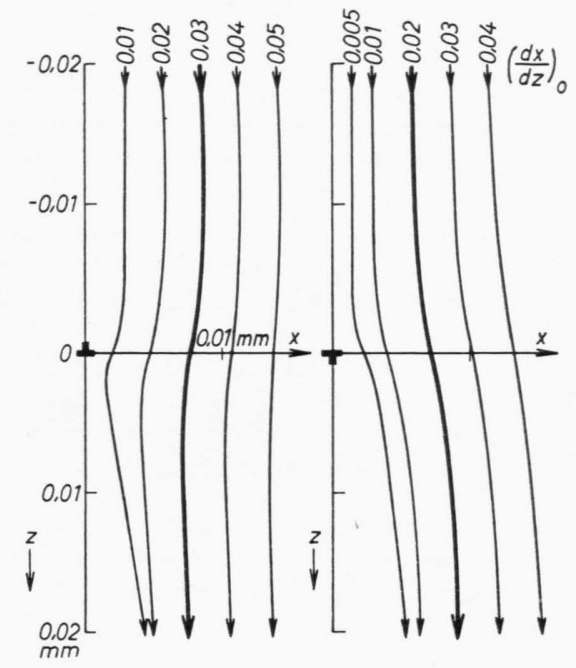

Abb. 2. Einzelheiten der Strahlwege in der Nähe der Versetzung. hören zu Wellenfeldern, die wegen des Bormann-Effektes anomal schwach absorbiert werden. Die Skizzen in den oberen Teilen der Abbildungen geben die Lage der überschüssigen Atomebene an, die an der Versetzungslinie endet. Man sieht, daß trotz des unterschiedlichen Kurvenverlaufs in den beiden Fällen, die gesamte Wirkung der Versetzung auf die Strahlen stets abstoßend ist. Die Strahlen, die der Versetzungslinie sehr nahe kommen, werden stärker abgebeugt. Infolgedessen entsteht hinter der Versetzung ein Schattenbereich, der durch die Enveloppe der Strahlen (Kaustik) begrenzt wird. In diesen Schattenbereich kann kein Strahl eindringen.

Abb. 2 zeigt die Details des Strahlengangs in der Nähe von Versetzungen, und zwar im realen Maßverhältnis zwischen $x$ und $z$. Die Strahlen, die durch dicke Linien gekennzeichnet sind, gelangen an die Schattenkante auf der Austrittsfläche (s. Abb. 1). Man sieht, daß diese Strahlen ziemlich weit von der Versetzung entfernt bleiben (Minimalabstand etwa $8 \mu$ ) und verhältnismäßig schwach gebogen sind. Man kann deshalb vermuten, daß die Methode von Penning und Polder für diese Strahlen noch eine gute Näherung gibt.

Die Strahlen, die noch näher an die Versetzung herankommen, erleiden stärkere Verbiegungen. Zur Berechnung der Wege dieser Strahlen sind wahrscheinlich höhere Näherungen der Theorie erforderlich. Sie spielen aber sicher keine Rolle für die Entstehung der Schattenkante auf der Austrittsfläche.

Unsere Resultate zeigen, daß die Schattenabbildung zumindest qualitativ durch die Penning-Poldersche Theorie gedeutet werden kann. Für die Berechnung der Intensitätsverteilung in der Nähe der Schattenkanten, über die später berichtet wird, sind die höheren Näherungen der Theorie des Verfassers anzuwenden.

\section{Magnetostriktion und Koerzitivfeldstärke einiger Fe-Si-Cr- und Fe-Si-Co-Legierungen}

\author{
Von Kurt-Hans v. Klitzing *
}

Mitteilung aus der Physikalisch-Technischen Bundesanstalt (Z. Naturforschg. 18 a, 1011-1012 [1963]; eingegangen am 13. Juli 1963)

Nachstehend werden einige bereits vor Jahren im Zuge einer größeren Untersuchung erhaltene Meßergebnisse an ternären $\mathrm{Fe}$-Si-Legierungen mitgeteilt. Durch sie wird experimentell bestätigt, daß man bei konstantem Eisengehalt von 75 At.-Proz. - ausgehend von der Verbindung $\mathrm{Fe}_{3} \mathrm{Si}$ - analog den Fe-Si-Al-Legierungen vom Typ Sendust ${ }^{1,2}$ - mit fortschreitender Substitution des Si auch durch andere Zusätze (an Stelle von $\mathrm{Al}$ ), wie Co oder $\mathrm{Cr}$, eine Vorzeichenumkehr der Magnetostriktion und ein relatives Minimum der Koerzitivfeldstärke erreichen kann.

\footnotetext{
* Braunschweig.

1 H. Masumoto, Sci. Rep. Tôhoku K. Honda Anniversary, Vol. 1936, p. 389.
}

Die Legierungen wurden im Hochfrequenzofen aus einem Ausgangseisen mit $0,2 \% \mathrm{C}$ erschmolzen und $\mathrm{zu}$ $6 \mathrm{~mm}$ dicken Rundstäben vergossen, danach bei $1000{ }^{\circ} \mathrm{C}$ im Vakuum 2 Stunden lang geglüht und langsam im Ofen abgekühlt. Die Magnetostriktion wurde mit Hilfe eines Drehspiegels (Röllchenmethode) gemessen, die Koerzitivfeldstärke mit einem astatischen Magnetometer.

Von den erhaltenen Ergebnissen gibt Abb. 1 zunächst Meßwerte der Längsmagnetostriktion binärer Fe-Si-Legierungen bei einer äußeren Feldstärke von $280 \mathrm{~A} / \mathrm{cm}$ wieder. Die miteingetragenen früheren Meßwerte von Schulze ${ }^{3}$ geben den Anschluß zu niedrigen $\mathrm{Si}$-Konzentrationen. In der Nähe der Verbindung $\mathrm{Fe}_{3} \mathrm{Si}$ konnten wegen der hier besonders hohen Sprödigkeit und Rissigkeit der Proben keine genauen Werte erhalten werden. Auf die Eintragung von Meßpunkten wurde deshalb an dieser Stelle verzichtet. - In Abb. 2

2 A. S. Zaimovsky u. I. P. Selissky, J. Phys. (Moskau) 4, 563 [1941].

3 A. Schulze, Z. Phys. 52, 490 [1928]. 


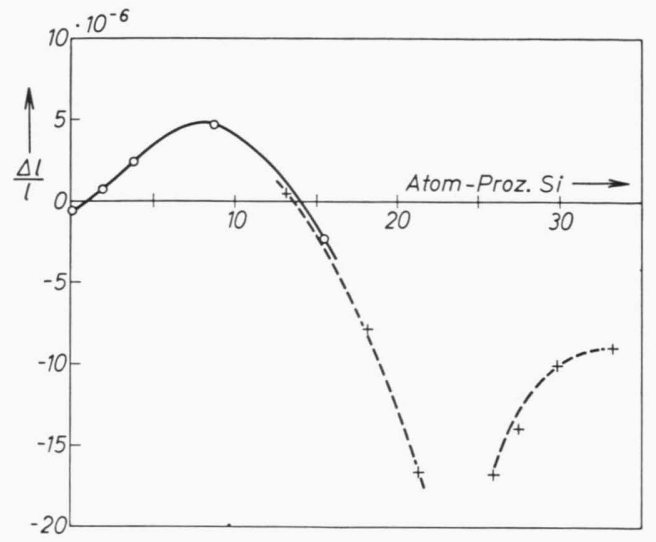

Abb. 1. Längsmagnetostriktion $\Delta l / l$ der $\mathrm{Fe}-\mathrm{Si}$-Legierungen. -O- Meßwerte nach Schulze ${ }^{3},--+--$ eigene Messungen.

finden sich die Werte der Magnetostriktion der ternären Legierungen, in Abb. 3 die der Koerzitivfeldstärke. Wie man sieht, liegt sowohl für Co- wie für Cr-Zusatz ein relatives Minimum der Koerzitivfeldstärke in der Nähe derjenigen Zusammensetzung, bei der die Ma-

${ }^{4}$ J. J. Went: in J. L. Snoek, New Developments in Ferromagnetic Materials, Elsevier Publ. Co., Inc. Amsterdam 1949, S. 19.

\section{Thermomagnetische Untersuchungen an Eisencarbiden}

Von Ph. Dünner und S. MülLer

Institut für theoretische Physik der Universität zu Köln, Abteilung für Metallphysik

(Z. Naturforschg. 18 a, 1012-1013 [1963]; eingeg. am 19. August 1963)

An einer martensitischen $\mathrm{Fe}-\mathrm{C}$-Legierung mit der in der Tabelle angegebenen Zusammensetzung wurden magnetische Sättigungsmessungen mit Hilfe einer automatisierten ferromagnetischen Waage nach LANGE und $\mathrm{M}_{\mathrm{ATHIEU}}{ }^{1}$ vorgenommen. Gemäß der Lage der PhasenCurie-Punkte in den Sättigungs-Temperatur-Kurven (S-T-Kurven) können die während des Anlassens dieser abgeschreckten $\mathrm{Fe}-\mathrm{C}$-Legierung auftretenden Phasen identifiziert werden.

\begin{tabular}{|c||c|c|c|c|c|c|}
\hline Elemente & $\mathrm{C}$ & $\mathrm{Mn}$ & $\mathrm{O}$ & $\mathrm{N}$ & $\mathrm{Si}$ & $\mathrm{Fe}$ \\
\hline Gew.-Proz. & 0,64 & 0,01 & 0,004 & 0,003 & Spuren & Rest \\
\hline
\end{tabular}

Tab. 1. Chemische Zusammensetzung des untersuchten Probenmaterials.

Nach dem Martensit-Zerfall und erfolgter RestAustenit-Umwandlung bei $190{ }^{\circ} \mathrm{C}$ Anlaßtemperatur

1 H. Lange, R. Kohlhaas u. S. Müller, Arch. Eisenhüttenwes. 34, 555 [1963].

2 E. Lehrer, Z. Elektrochem. 36, 460 [1930].
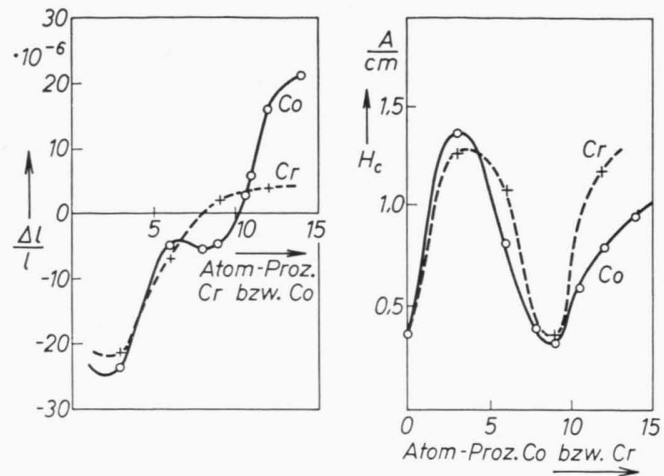

Abb. 2. Längsmagnetostriktion $\Delta l / l$ der Legierungen mit $75 \mathrm{At}$.Proz. Fe, $(25-x)$ At.-Proz. Si und $x$ At.-Proz. Co oder Cr.
Abb. 3. Koerzitivfeldstärken $H_{\mathrm{c}}$ der Legierungen entsprechend Abb. 2. gnetostriktion durch Null geht. Es ist anzunehmen, daß bei reinem, C-freiem Ausgangsmaterial noch wesentlich niedrigere $H_{\mathrm{c}}$-Werte erreicht werden können. Hohe Werte der Anfangspermeabilität, wie im System $\mathrm{Fe}-\mathrm{Si}-\mathrm{Al}$, sind indessen bei Fe-Si-Co-Legierungen nicht zu erwarten, weil nach $W_{\text {ENT }}{ }^{4}$ in diesem System die Minima der Kristallenergie nirgends mit einer Nullstelle der Magnetostriktion zusammenfallen.

liegt als Ausgangszustand für eine gesonderte Untersuchung der sogenannten 3. Temperstufe ein Phasengemisch von hexagonalem $\varepsilon$-Eisencarbid, schwach tetragonalem Rest-Martensit (low carbon martensite) und Ferrit oder sekundärem Martensit vor. Dieses Phasengemisch wurde bei drei verschiedenen Anlaßtemperaturen, $350{ }^{\circ} \mathrm{C}, 450{ }^{\circ} \mathrm{C}$ und $550{ }^{\circ} \mathrm{C}$, untersucht. Die S-T. Kurven sind jeweils vor und nach jeder Temperung von Zimmertemperatur bis zur Anlaßtemperatur aufgenommen worden. Die erste Erhitzungskurve weist einen Curie-Punkt bei $320^{\circ} \mathrm{C}$ auf, der in Analogie zu Ergebnissen an Eisennitriden von LeHRER ${ }^{2}$ einem hexagonalen $\varepsilon$-Eisencarbid mit einer chemischen Zusammensetzung von $\sim \mathrm{Fe}_{2} \mathrm{C}$ zuzuschreiben ist, wie dies CRAngle und Sucкsмiтн ${ }^{3}$ ebenfalls aus ihren Messungen folgern. Die nach jeweils steigender Temperatur gemessenen Abkühlungskurven (S-T-Kurven) weisen zunächst zwei neue Curie-Punkte auf, während derjenige bei $320^{\circ} \mathrm{C}$ nicht mehr aufzufinden ist. Einer der beiden neuen Curie-Punkte liegt bei $255^{\circ} \mathrm{C}$. Seiner Lage nach ist er einem Zwischencarbid mit einer chemischen Zusammensetzung nahe der des komplexen Percarbids $\mathrm{Fe}_{20} \mathrm{C}_{9}$ zuzuordnen. Dieses Zwischencarbid wird im folgenden in Anlehnung an die Bezeichnungsweise von Apajev ${ }^{4}$ als $\chi$-Carbid bezeichnet. Der andere CurIE-Punkt verschiebt sich, wie Abb. 1 zeigt, von oberhalb $250{ }^{\circ} \mathrm{C}$ mit zuneh-

3 J. Crangle u. W. Sucksmith, J. Iron Steel Inst. (London) 168, 141 [1951].

${ }^{4}$ B. A. Apajev, Fiz. Metal. i Metalloved. (engl. Übers.) 9, 70 [1960]. 